\title{
Quali sono le ultime novità in tema di terapia marziale?
}

\author{
Andrea Cavalli, Giuseppe Pontoriero
}

\author{
S.C. Nefrologia e Dialisi, Ospedale “Alessandro Manzoni”, Lecco
}

\begin{abstract}
WHAT'S THE LATEST ON IRON THERAPY?
Abstract. Anemia is a common complication of chronic kidney disease (CKD) and is usually treated with erythropoiesis-stimulating agents (ESA) and iron supplementation.

Endovenous iron therapy has been recently ruled (and limited) by both the European and Italian medicines agencies due to serious, despite rare, adverse events.

A recent analysis of data from the Dialysis Outcomes and Practice Patterns Study (DOPPS) has shown an association between high endovenous iron therapy (more than $300 \mathrm{mg} / \mathrm{month}$ ) and increased mortality in patients with hemoglobin levels higher than $10 \mathrm{~g} / \mathrm{dL}$. The new molecules available on the market should have a better safety profile and several advantages related to the possibility of administering a high iron dose in a single infusion.

Iron supplementation through the dialysate and through new phosphate binders demonstrates the great scientific interest in this important field, also to the advantage of our CKD patients.
\end{abstract}

Key words: Iron therapy, Endovenous iron, DOPPS, Mortality, Adverse events

Conflict of interest: None.

Financial support: None.

Accettato: 17 Settembre 2014

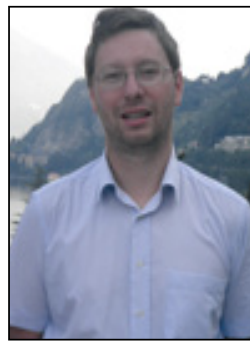

Andrea Cavalli
L'anemia è una frequente complicanza dell'insufficienza renale cronica (IRC), presente in circa il $50 \%$ dei pazienti con IRC agli stadi 3-5 e in percentuali più elevate nei soggetti dializzati (1). Oggigiorno l'utilizzo di agenti stimolanti l'eritropoiesi (ESA) e la supplementazione marziale sono gli strumenti principali per trattare l'anemia nei pazienti con IRC.

Nei pazienti con IRC, la terapia marziale non serve solo a correggerne la carenza, ma è anche una fondamentale terapia di supporto in corso di ESA, permettendone addirittura una riduzione del fabbisogno (2). I risultati dello studio TREAT (3), quelli del trial di Stancu (4), in cui si evidenziava che la terapia con ferro per via endovenosa era in grado di aumentare $i$ livelli di emoglobina anche in soggetti senza carenza marziale, e il position paper redatto dal gruppo European Renal Best Practice (ERBP) nel 2010 (5) hanno recentemente sottolineato il ruolo della terapia marziale.

Tuttavia, è noto come da una parte le formulazioni tradizionali per la somministrazione marziale per via orale possano frequentemente essere mal tollerate e/o poco efficaci, mentre, dall'altra, quelle per via endovenosa (e.v.) possano causare rare ma pericolose reazioni anafilattiche, severa ipotensione o la necessità di essere somministrate a piccole dosi ripetute con lunghi tempi di infusione per evitarne gli effetti indesiderati. I suddetti eventi si possono ricondurre a un aumentato stress ossidativo, a infiammazione e a disfunzione endoteliale e immune (6).

Il recente comunicato dell'agenzia europea per i medicinali (EMA) (7), ribadito nell'Ottobre del 2013 anche dall'agenzia italiana del farmaco (AIFA) (8), derivato da severi eventi avversi verificatisi in pazienti gravide che avevano ricevuto ferro per via parenterale, ha sottolineato come tale terapia necessiti di essere praticata solo in strutture in cui sia immediatamente disponibile del personale qualificato in grado di valutare e gestire reazioni anafilattiche/anafilattoidi e apparecchiature rianimatorie. Questo ha determinato gravi ricadute anche in campo Nefrologico-Dialitico, sia dal punto di vista legale (e burocratico), con la necessità di ottenere un consenso informato da parte del paziente in occasione della suddetta terapia, sia dal punto di vista clinico (e pratico), in quanto nei Centri Dialisi ad assistenza limitata non sono sempre presenti i requisiti richiesti 


\section{TABELLA I - MESSAGGI/INDICAZIONI DELL'AGENZIA ITALIANA DEL FARMACO IN MERITO ALLA SOMMINISTRAZIONE DI FERRO PER VIA ENDOVENOSA}

1. Il rischio di ipersensibilità è maggiore nei pazienti con allergie note o affetti da patologie infiammatorie o del sistema immunitario (per esempio, lupus eritematoso sistemico).

2. La somministrazione può avvenire solo quando sia immediatamente disponibile del personale qualificato, in grado di valutare e gestire reazioni anafilattiche/ anafilattoidi e apparecchiature di rianimazione.

3. I medici prescriventi devono informare i pazienti del rischio di ipersensibilità prima di ogni somministrazione e dei sintomi correlati, oltre che raccomandare di contattare con urgenza il medico in caso di reazioni avverse.

4. I pazienti devono essere strettamente monitorati per la comparsa di eventuali segni di ipersensibilità durante e per almeno 30 minuti dopo ogni somministrazione di ferro per via endovenosa.

5. Le dosi di prova non sono più raccomandate e si deve usare cautela con ogni dose, anche se le somministrazioni precedenti sono state ben tollerate.

6. Le reazioni avverse si possono verificare anche dopo una precedente somministrazione ben tollerata (compresa una dose di prova negativa).

TABELLA II - MESSAGGI DERIVANTI DALLO STUDIO DI BAILIE (9)

1. Dose media ricevuta di ferro e.v. pari a $252 \mathrm{mg} / \mathrm{mese}$.

2. In Europa, le formulazioni di ferro e.v. più utilizzate sono: ferro saccarosio (60\%), ferro gluconato (24\%) e ferro polimaltoso (11\%).

3. Dosi di ferro e.v. di 300-399 mg/mese e $>400 \mathrm{mg} / \mathrm{mese}$ si associano a un aumento della mortalità per tutte le cause del $13 \%$ e del $18 \%$ rispettivamente.

4. La mortalità è maggiore solo se l'emoglobina è superiore a $10 \mathrm{~g} / \mathrm{dL}$ (ed è ancora più marcata se $>12 \mathrm{~g} / \mathrm{dL}$ ).

5. Associazione riscontrata anche tra dose di ferro e.v. $>300 \mathrm{mg} / \mathrm{mese}$ e mortalità per cause cardio-vascolari.

6. Dosi di ferro e.v. $>6 \mathrm{mg} / \mathrm{kg}$ si associano a un'aumentata mortalità per tutte le cause e per cause cardio-vascolari (rispettivamente $26 \%$ e $35 \%$ ).

7. Dosi di ferro e.v. $>300 \mathrm{mg} / \mathrm{mese}$ si associano anche a un rischio di ospedalizzazione più elevato $(+12 \%)$.

dall'EMA, con la conseguenza di dover sospendere la terapia marziale per via endovenosa in numerosi pazienti (Tab. I). Tuttavia, un recente articolo pubblicato su Kidney International (9), di cui vogliamo occuparci nella nostra rubrica $T N \& D$ Journal Club, ha aggiunto ulteriori elementi di discussione in merito alla terapia con ferro. Bailie ha analizzato i dati di 32.435 pazienti emodializzati che hanno partecipato alle fasi 2, 3 e 4 dello studio Dialysis Outcomes and Practice Patterns Study (DOPPS) di cui fossero disponibili i dati prescrittivi della terapia marziale e.v. (Tab. II). Lo studio DOPPS aveva peraltro già recentemente sottolineato la variabilità geografica della prescrizione della terapia marziale, oltre a un progressivo diffuso aumento della dose di ferro somministrata nel corso degli ultimi 10-15 anni (10).

Nell'articolo di Bailie, la dose totale di ferro e.v. somministrata nei 4 mesi di osservazione è stata espressa come dose media per mese di terapia (mg/mese): il 32\% dei soggetti non ha ricevuto supplementazione marziale, il $10 \%$ ha ricevuto $1-99 \mathrm{mg}$ / mese, il 19\% 100-199 mg/mese, il 17\% 200-299 mg/mese, il $6 \% 300-399 \mathrm{mg} / \mathrm{mese}$ e il $15 \%$ oltre $400 \mathrm{mg} / \mathrm{mese}$. La dose media ricevuta era di $252 \mathrm{mg} / \mathrm{mese}$ e quella più frequente era pari a $200 \mathrm{mg} / \mathrm{mese}(12 \%$ dei casi). Le diverse tipologie di ferro somministrato e.v. variavano anche a seconda dell'area geografica: in Europa si utilizzava ferro saccarosio (60\%), gluconato $(24 \%)$ e polimaltoso (11\%) (9).

Le più alte dosi di ferro e.v. venivano somministrate in soggetti con ridotta età dialitica e portatori di catetere venoso centra- le come accesso vascolare per emodialisi, con più alto indice di massa corporea, alta prevalenza di scompenso cardiaco, neoplasie, sanguinamento gastroenterico e vasculopatia periferica e con più bassi valori di saturazione della transferrina e in terapia con maggiori dosaggi di ESA (9).

La possibile associazione tra supplementazione marziale e.v. e mortalità è stata valutata confrontando le diverse dosi di ferro e.v. rispetto al dosaggio più comune (100-199 mg/mese). Le classi $0,1-99$ e $200-299 \mathrm{mg} / \mathrm{mese}$ presentavano sovrapponibili rischi relativi (HR) per morte per tutte le cause, mentre i più elevati dosaggi erano associati a una maggiore mortalità. In particolare, i dosaggi 300-399 mg/mese e >400 mg/ mese presentavano rispettivamente un HR di 1.13 (intervallo di confidenza al 95\% (IC 95\%): 1.00-1.27) e di 1.18 (IC 95\%: 1.07-1.30), corrispondenti a una mortalità aumentata del $13 \%$ e del $18 \%$. Anche dicotomizzando il dosaggio di ferro e.v. (superiore o inferiore a $300 \mathrm{mg} / \mathrm{mese}$ ) si confermava una maggiore mortalità associata alle dosi più elevate (HR 1.12, IC 95\%: 1.04-1.20), che persisteva dopo aver "aggiustato" per variabili come comorbidità, livelli di albumina, emoglobina, assetto marziale e terapia con ESA (9).

Veniva evidenziato come l'associazione di terapia con ferro e.v. a dosaggi superiori a $300 \mathrm{mg} / \mathrm{mese}$ con una più elevata mortalità fosse persistente durante le fasi DOPPS 2-4 in Europa e 3-4 negli Stati Uniti. Inoltre, questo risultato era indipendente dall'anzianità dialitica, dall'età dei pazienti e dai livelli di proteina $\mathrm{C}$ reattiva e di saturazione della tran- 
sferrina e della ferritina, così come dalla tipologia di ferro o dalla frequenza di somministrazione. Tuttavia, la prognosi si rivelava peggiore solo in quei soggetti con livelli di emoglobina compresa tra 10 e 12 g/dL (HR 1.12, IC 95\%: 1.02-1.22) e superiore a $12 \mathrm{~g} / \mathrm{dL}$ (HR 1.25, IC 95\%: 1.10-1.41), ma non in pazienti con emoglobina inferiore a $10 \mathrm{~g} / \mathrm{dL}$ (HR 0.94 , IC 95\%: 0.80-1.10) (9).

Si evidenziava un'associazione anche tra dose di ferro e.v. superiore a $300 \mathrm{mg} / \mathrm{mese}$ e aumentata mortalità di origine cardiovascolare, mentre non si raggiungeva la significatività statistica per quanto riguarda la morte da causa infettiva o di altra natura, forse anche per la bassa numerosità di tali eventi (9).

La normalizzazione della dose di ferro e.v. per il peso corporeo ha mostrato un'associazione con un'aumentata mortalità per tutte le cause e per cause cardio-vascolari (rispettivamente $26 \%$ e $35 \%$ ) quando si superavano i $6 \mathrm{mg} / \mathrm{kg}$ al mese di supplementazione marziale e.v. (9).

Anche il rischio di ospedalizzazione era più elevato quando la dose di ferro e.v. era superiore a $300 \mathrm{mg} / \mathrm{mese}$ (HR 1.12, IC 95\%: 1.07-1.18) (9).

Come gli Autori stessi riconoscono, il loro articolo presenta delle limitazioni. Infatti, oltre a non essere stato valutato l'effetto della terapia marziale e.v. nel lungo termine, i dati presentati sono derivati da uno studio osservazionale e quindi non possono stabilire un nesso di causalità, ma solo generare delle ipotesi che necessitano poi di essere verificate in adeguati trial clinici randomizzati e controllati (9).

Due precedenti studi osservazionali $(11,12)$ condotti su ampie popolazioni di emodializzati (rispettivamente oltre 32.000 e oltre 58.000) hanno valutato lo stesso outcome analizzato da Bailie. Mentre il primo (11), più datato, non ha mostrato differenze tra "alte" e "basse" dosi di ferro e.v., il secondo (12) ha riportato un'aumentata mortalità per tutte le cause e per cause cardio-vascolari in coloro che ricevevano un quantitativo di ferro e.v. maggiore di $400 \mathrm{mg} / \mathrm{mese}$ rispetto a quanti non venivano supplementati. Un'ulteriore e successiva analisi su circa 270.000 emodializzati americani condotta tra il 1999 e il 2007 ha evidenziato un'associazione tra elevata mortalità e maggiori dosi di ferro e.v. solo in caso di ematocrito superiore al 36\%, mentre, se l'ematocrito era inferiore al 30\%, non emergeva tale dato (13).

Ma come correlare il ferro e.v. con un'aumentata mortalità? È stato ipotizzato che la terapia marziale e.v. possa contribuire alla severa prognosi associata all'IRC e in particolare che lo stress ossidativo ad essa secondario determini la trasformazione delle cellule muscolari lisce dei vasi in cellule a fenotipo osteoblastico, responsabili della formazione di calcificazioni vascolari (6). Occorre, però, sottolineare come, in seguito alla somministrazione di composti a base di ferro, la quantità di elemento libero rilasciata in circolo (e, quindi, potenzialmente responsabile di stress ossidativo) dipenda dalla molecola impiegata.

Anche per questo motivo, sono state recentemente sviluppate nuove molecole per la supplementazione marziale, dotate di un elevato peso molecolare, teoricamente in grado di causare minori danni ossidativi e tissutali rispetto alle più vecchie formulazioni (14) e dotate pertanto di un migliore profilo di sicurezza, che permette loro di essere somministrate a dosi più elevate e in singola somministrazione. Questa caratteristica è
TABELLA III - NUOVE MOLECOLE PER LA SUPPLEMENTAZIONE MARZIALE E.V.

\author{
1. Ferro carbossimaltosio ("Ferinject") \\ 2. Ferumoxitolo ("Rienso") \\ 3. Ferro isomaltoside 1000 ("Diafer")
}

certamente di grande rilevanza clinica, soprattutto per quei pazienti in fase pre-dialitica necessitanti di terapia marziale e.v. in cui occorre preservare il patrimonio venoso in vista dell'allestimento dell'accesso vascolare per emodialisi e che, invece, rischierebbe di essere danneggiato da ripetute punture venose. Inoltre si otterrebbe una riduzione del numero di accessi ospedalieri, oltre che del carico infermieristico, nella gestione di questi pazienti.

Recenti autorevoli review circa le attuali raccomandazioni nel trattamento dell'anemia secondaria a IRC e le nuove terapie emergenti $(15,16)$ riportano le caratteristiche salienti di questi composti, di cui vogliamo parlare in breve (Tab. III).

Il ferro carbossimaltosio ("Ferinject", Vifor Pharma) contiene ferro in stato ferrico stabile, sotto forma di complesso di un nucleo di ferro-idrossido polinucleare con un ligando carboidrato. È stato testato in più popolazioni di soggetti con anemia sideropenica (IRC, malattie infiammatorie croniche, puerpere) e ha evidenziato una non-inferiorità rispetto alla supplementazione orale o con ferro saccarosio, oltre a una maggiore rapidità nel correggere l'anemia e nell'aumentare i livelli di ferritina $(17,18)$. Il farmaco è utilizzato in molti Paesi europei già dal 2007 e può essere utilizzato sia in fase conservativa sia in pazienti dializzati, quando i preparati a base di ferro per via orale sono inefficaci o non possono essere usati. La dose singola massima di ferro carbossimaltosio è pari a $1000 \mathrm{mg}$, non somministrabile più frequentemente di una volta alla settimana, ma, nei soggetti in emodialisi, non si può superare una dose massima giornaliera di $200 \mathrm{mg}$.

Il ferumoxitolo ("Rienso", AMAG Pharmaceuticals) è un complesso carboidrato a base di ferro colloidale, sviluppato inizialmente come mezzo di contrasto per la risonanza magnetica, approvato negli USA nel 2009 e recentemente anche dall'EMA. Può essere somministrato a pazienti con qualunque grado di IRC (19) e alla dose di $510 \mathrm{mg}$ in pochi secondi. In un recente studio randomizzato (20) il ferumoxitolo si è dimostrato di pari efficacia al ferro saccarosio, anche dal punto di vista della sicurezza.

Il ferro isomaltoside 1000 ("Diafer", Pharmacosmos) è un composto oligosaccaridico. E stato valutato in più ambiti di anemia sideropenica e ha mostrato un'adeguata efficacia anche in pazienti con IRC. È disponibile in Europa dal 2010 e in Italia dal Luglio $2013(15,16)$.

Il desiderio di cercare di ottimizzare e rendere sempre più sicura la supplementazione marziale e.v., anche nel paziente emodializzato, è diffuso e attivo su tutti i fronti possibili (Tab. IV). Ne è un chiaro esempio l'innovativo programma della Rockwell Medical, volto alla valutazione del composto Triferic $^{\mathrm{TM}}$, il quale viene somministrato tramite il dialisato, con l'obiettivo di rimpiazzare quei 5-7 $\mathrm{mg}$ di ferro persi 


\section{TABELLA IV - POSSIBILI MODALITÀ DI SUPPLEMENTA- ZIONE MARZIALE NEL PAZIENTE EMODIA- LIZZATO}
1. Per os
2. Per via endovenosa
3. Attraverso i chelanti del fosforo
4. Nel dialisato

durante ogni seduta emodialitica. Triferic ${ }^{\mathrm{TM}}$ viene aggiunto nel concentrato di bicarbonato di sodio prima della sessione di dialisi e successivamente mischiato al dialisato. Una volta superato il filtro, entra nel circolo ematico, si lega alla transferrina e viene condotto nel midollo osseo, così come avviene per il ferro di origine alimentare (21).

In realtà, già nel 1999, Gupta et al. (22) avevano dimostrato che la somministrazione marziale (in particolare, pirofosfato ferrico) attraverso il dialisato poteva rappresentare una sicura ed efficace alternativa al ferro destrano per via endovenosa.

Nel Maggio del 2013, i dati dello studio PRIME, trial multicentrico, randomizzato e controllato, condotto per 9 mesi in 103 pazienti emodializzati, hanno mostrato come l'utilizzo di dialisato addizionato di Triferic ${ }^{\mathrm{TM}}$ rispetto a quello tradizionale (permettendo una terapia con ferro saccarato e.v. in caso di carenza marziale) consenta di ridurre del 35\% il consumo di ESA (e del 74\% nei pazienti iporesponsivi all'ESA). Veniva inoltre evidenziato come non vi fosse un aumento né dello stress ossidativo né dei livelli di epcidina e come non vi fosse un accumulo di ferro (23). Da qualche mese è terminato uno studio di fase III (CRUISE 1) che ha valutato la sicurezza di Triferic $^{\mathrm{TM}}$ in oltre 700 soggetti emodializzati (24). Inoltre, esistono e, probabilmente, a breve saranno in commercio anche in Italia chelanti del fosforo a base di ferro che potrebbero contribuire a una migliore gestione della terapia marziale. Infatti, il citrato ferrico si è recentemente dimostrato efficace e sicuro nella riduzione della fosforemia, ma anche capace di aumentare le scorte marziali e di ridurre il fabbisogno di ferro e.v. e di ESA (a parità di valori di emoglobina, ovviamente) (25).

Attualmente è ben chiaro, quindi, come la discussione in merito alla terapia marziale (non solo per via endovenosa) sia caratterizzata da un grande fermento, che, fino a qualche anno fa, in pochi avrebbero immaginato. Occorrerà imparare a scegliere la migliore soluzione possibile per ogni paziente, in quanto sono le caratteristiche peculiari del singolo che devono guidarci nella corretta prescrizione... insomma, come sempre nel nostro lavoro, bisogna ragionare!

\section{Riassunto}

L'anemia è una frequente complicanza dell'insufficienza renale cronica (IRC), per il cui trattamento gli strumenti principali sono gli agenti stimolanti l'eritropoiesi (ESA) e la supplementazione marziale.

La terapia marziale per via endovenosa è stata ultimamente oggetto di regolamentazione (e limitazione) da parte delle agenzie del farmaco europea e italiana, in quanto associata a gravi (anche se rari) eventi avversi.

Una recente analisi di dati provenienti dallo studio Dialysis Outcomes and Practice Patterns Study (DOPPS) ha mostrato un'associazione tra elevata supplementazione marziale per via endovenosa (superiore a $300 \mathrm{mg} / \mathrm{mese}$ ) e aumentata mortalità, nei pazienti con valori di emoglobina superiori a $10 \mathrm{~g} / \mathrm{dL}$.

Le nuove molecole disponibili in commercio dovrebbero presentare un migliore profilo di sicurezza e vantaggi legati alla possibilità di somministrare elevate dosi di ferro in un'unica occasione.

La possibilità di fornire supplementazione marziale attraverso il dialisato e con l'assunzione di nuovi chelanti del fosforo dimostra il grande fervore scientifico che oggi caratterizza questo importante ambito clinico, anche per i nostri pazienti con IRC.

Parole chiave: Terapia marziale, Ferro per via endovenosa, DOPPS, Mortalità, Eventi avversi

Dichiarazione di conflitto di interessi: Gli Autori dichiarano di non avere conflitto di interessi.

Contributi economici agli Autori: Gli Autori dichiarano di non aver ricevuto sponsorizzazioni economiche per la preparazione dell'articolo.

Indirizzo degli Autori:

Prof. Giuseppe Pontoriero

S.C. Nefrologia e Dialisi

Ospedale "Alessandro Manzoni"

Via dell'Eremo 9/11

23900 Lecco

g.pontoriero@ospedale.lecco.it 


\section{Bibliografia}

1. McClellan WM, Aronoff SL, Bolton WK. The prevalence of anaemia in patients with chronic kidney disease. Curr Med Res Opin. 2004; 20: 1501-10.

2. Fishbane S, Frei GL, Maesaka J. Reduction in recombinant human erythropoietin doses by the use of chronic intravenous iron supplementation. Am J Kidney Dis. 1995; 26: 41-6.

3. Pfeffer MA, Burdmann EA, Chen CY, et al. A trial of darbepoetin alfa in type 2 diabetes and chronic kidney disease. N Engl J Med. 2009; 361: 2019-32.

4. Stancu S, Barsan L, Stanciu A, Mircescu G. Can the response to iron therapy be predicted in anemic nondialysis patients with chronic kidney disease? Clin J Am Soc Nephrol. 2010; 5: 409-16.

5. Locatelli F, Aljama P, Canaud B, et al. Anaemia Working Group of European Renal Best Practice (ERBP). Target haemoglobin to aim for with erythropoiesis-stimulating agents: a position statement by ERBP following publication of the Trial to reduce cardiovascular events with Aranesp therapy (TREAT) study. Nephrol Dial Transplant. 2010; 25(9): 2846-50.

6. Vaziri ND. Epidemic of iron overload in dialysis population caused by intravenous iron products: a plea for moderation. Am J Med. 2012; 125: 951-2.

7. European medicines agency. Available from: www.ema.europa. eu/ema/index.jsp? curl=pages/news_and_events [Last accessed 26 February 2014].

8. Available from: http://www.agenziafarmaco.gov.it/it/content/ nota-informativa-importante-su-medicinali-contenenti-ferro-25102013 [Last accessed 10 September 2014].

9. Bailie GR, Larkina M, Goodkin DA, et al. Data from the Dialysis Outcomes and Practice Patterns Study validate an association between high intravenous iron doses and mortality. Kidney Int. 2014; advance online publication 30 July 2014.

10. Bailie GR, Larkina M, Goodkin DA, et al. Variation in intravenous iron use internationally and over time: the Dialysis Outcomes and Practice Patterns Study (DOPPS). Nephrol Dial Transplant. 2013; 28: 2570-9.

11. Feldman HI, Joffe M, Robinson B, et al. Administration of parenteral iron and mortality among hemodialysis patients. J Am Soc Nephrol. 2004; 15: 1623-32.

12. Kalantar-Zadeh K, Regidor DL, McAllister CJ, et al. Time- dependent associations between iron and mortality in hemodialysis patients. J Am Soc Nephrol. 2005; 16: 3070-80.

13. Brookhart MA, Schneeweiss S, Avorn J, et al. Comparative mortality risk of anemia management practices in incident hemodialysis patients. JAMA. 2010; 303: 857-64.

14. Bailie GR, Schuler C, Leggett RE, et al. Oxidative effect of several intravenous iron complexes in the rat. Biometals. 2013; 26 (3): 473-8.

15. Del Vecchio L, Locatelli F. Anemia in chronic kidney disease patients: treatment recommendations and emerging therapies. Expert Rev Hematol. 2014; 7 (4): 495-506.

16. Macdougall IC, Geisser P. Use of intravenous iron supplementation in chronic kidney disease: an update. Iran J Kidney Dis. 2013; 7 (1): 9-22.

17. Lyseng-Williamson KA, Keating GM. Ferric carboxymaltose: a review of its use in iron-deficiency anaemia. Drugs. 2009; 69 (6): 739-56.

18. Covic A, Mircescu G. The safety and efficacy of intravenous ferric carboxymaltose in anaemic patients undergoing haemodialysis: a multi-centre, open-label, clinical study. Nephrol Dial Transplant. 2010; 25 (8): 2722-30.

19. Singh A, Patel T, Hertel J, et al. Safety of ferumoxytol in patients with anemia and CKD. Am J Kidney Dis. 2008; 52 (5): 907-15.

20. Macdougall IC, Strauss WE, McLaughlin J, et al. A randomized comparison of ferumoxytol and iron sucrose for treating iron deficiency anemia in patients with CKD. Clin J Am Soc Nephrol. 2014; 9 (4): 705-12.

21. http://www.rockwellmed.com/bio-pharma-soluble-ferric-pyrophosphate-clinical-development.htm

22. Gupta A, Amin NB, Besarab A, et al. Dialysate iron therapy: infusion of soluble ferric pyrophosphate via the dialysate during hemodialysis. Kidney Int. 1999; 55 (5): 1891-8.

23. Abstracts presented at the $51^{\text {st }}$ ERA-EDTA Congress in Amsterdam, May 31-June 3, 2014. "Triferic ${ }^{\mathrm{TM}}$ Does Not Induce Oxidative Stress in CKD-HD: The Prime Study" and "Triferic ${ }^{\mathrm{TM}}$ Does Not Increase Predialysis Hepcidin Levels or Iron Sequestration: The Prime Study".

24. http://clinicaltrials.gov/show/NCT01320202.

25. Lewis JB, Sika M, Koury MJ, et al. Ferric citrate controls phosphorus and delivers iron in patients on dialysis. J Am Soc Nephrol. 2014 Jul 24. Epub ahead of print. 